\title{
Statistically Robust Cognitive Radio Beamforming
}

\author{
Sudhir Singh*, Paul D. Teal ${ }^{\dagger}$, Pawel A. Dmochowski ${ }^{\dagger}$ and Alan J. Coulson* \\ * Industrial Research Limited, Lower Hutt, New Zealand \\ † School of Engineering and Computer Science, Victoria University of Wellington, Wellington, New Zealand \\ Email:\{s.singh,a.coulson\}@irl.cri.nz, \{paul.teal,pawel.dmochowski\}@ vuw.ac.nz
}

\begin{abstract}
We consider a cognitive radio (CR) network consisting of a secondary user transmitter (SU-Tx) equipped with multiple antennas and a secondary user receiver (SU-Rx) that share spectrum with multiple primary user transmitter (PUTx) and receiver (PU-Rx) pairs. We assume that the $C R$ has a loose cooperation with the primary network and therefore, only partial channel state information of each of the PU-Tx to PU-Rx and SU-Tx to each PU-Rx links is available. Furthermore, we assume that the SU-Tx to SU-Rx link CSI is imperfect, with the channel error modelled as additive Gaussian noise. Under these assumptions, we propose a new statistically robust CR beamformer where the total SU-Tx transmit power is minimised subject to $\mathrm{PU}-\mathrm{Rx}$ and SU-Rx outage probability constraints. We present expressions for PU-Rx and SU-Rx outage probabilities and formulate the robust beamformer optimisation problem as a convex semidefinite program (SDP). SU-Tx transmit power, PU-Rx signal-to-interference-and-noise ratio (SINR) and SU-Rx signal-to-noise (SNR) cumulative distribution functions (CDFs) are obtained through solution of our optimisation problem.
\end{abstract}

\section{INTRODUCTION}

A number of papers have appeared on various aspects of cognitive radio (CR) systems, including capacity limit dependence on channel state information (CSI) (see, eg., [1,2]). In an underlay CR system the secondary users (SUs) protect the primary user (PU) by regulating their transmissions to maintain the PU receiver interference below a defined threshold level. The limits on this received interference level at the PU receiver can be imposed by an average/peak constraint, or a minimum signal-to-interference-and-noise ratio (SINR). The advantage of using an SINR-based PU protection mechanism, as opposed to PU interference temperature based protection, is that it removes the constant interference threshold, thus benefiting the SUs when the PU link is strong.

Beamforming has been shown to significantly improve performance in conventional wireless systems [3-6]. Recently, there has been significant progress in the field of beamforming using convex optimisation techniques. In [7,8], the beamforming problem has been recast into tractable convex optimisation problems and efficiently solved using interior point methods [9]. In [3-8], the beamformers were designed under the assumption of full CSI. In practise, full CSI is seldom available. To this extent, several works have appeared on the design of robust cooperative beamformers based on worst case performance optimisation that assume deterministic upper bounds on the norm of the channel errors [10-13]. Unfortunately, solutions obtained through worst case approach can be overly conservative because the probability of worst case errors may be extremely low [14]. Since the wireless channel varies randomly, it makes more sense to consider a probabilistic model for the design of robust beamformers. Outage probability based statistically robust beamformers have been proposed in $[14,15]$.

Due to its advantages, beamforming has attracted much interest in CR research [16-20]. A typical spectrum sharing cognitive network consists of a SU transmitter (SU-Tx) and a SU receiver (SU-Rx) that coexist with a number of primary transmitters and receivers. One of the challenges of spectrum sharing is guaranteeing quality of service (QoS) to the PU. Generally the goals of the CR are not compatible with the goals of the PU. For instance, increasing SU power to increase SU capacity will tend to increase interference to the PU. Beamforming is seen as a way to alleviate some of these issues, since the SU can direct its power away from the PU receivers. Design of $\mathrm{CR}$ beamformers under the assumption of full channel state information (CSI) has been the subject of investigation in $[16,17]$. In $[18,19]$, the design of robust worst case CR beamformers under the assumption of partial CSI have been studied. Most recently, in [20], the design of a statistically robust CR beamformer under the assumption of imperfect CSI for a CR network with close cooperation with the primary network was addressed.

In this paper, we consider the scenario where there are multiple PU transmitter and receiver pairs coexisting with a SU-Tx and SU-Rx pair. We assume that the CR network has a loose cooperation with the primary network and, therefore, only mean channel powers of each of the PU transmitter (PUTx) to PU receiver (PU-Rx) and the SU-Tx to each PU-Rx links are available. Furthermore, we assume that the SU-Tx to SU-Rx link CSI is imperfect, with the channel error modelled as additive Gaussian noise. We propose a new statistically robust CR beamformer where the total SU-Tx transmit power is minimised subject to PU-Rx and SU-Rx outage probability constraints. We present expressions for PU-Rx and SU-Rx outage probabilities and formulate the robust beamformer optimisation problem as a convex semidefinite program (SDP). SU-Tx transmit power, PU-Rx SINR and SU-Rx signal-tonoise (SNR) cumulative distribution functions (CDFs) are obtained through solution of our optimisation problem.

Notation: Upper (lower) bold face letters are used for matrices (vectors); $(\cdot)^{*},(\cdot)^{T},(\cdot)^{H}, \mathbb{E}\{\cdot\}$ and $\|\cdot\|$ denote complex conjugate, transpose, Hermitian transpose, expectation and Euclidean norm for vectors or Frobenius norm for matrices, respectively. $|\cdot|^{2}$ denotes the magnitude squared operator for scalars and elementwise magnitude squared for vectors. $\operatorname{tr}(\cdot), \mathcal{C}^{R \times 1}, \mathcal{C}^{R \times R}, \Re\{\cdot\}$ and $\Im\{\cdot\}$ denote the matrix trace operator, space of $R \times 1$ vectors with complex entries, space 


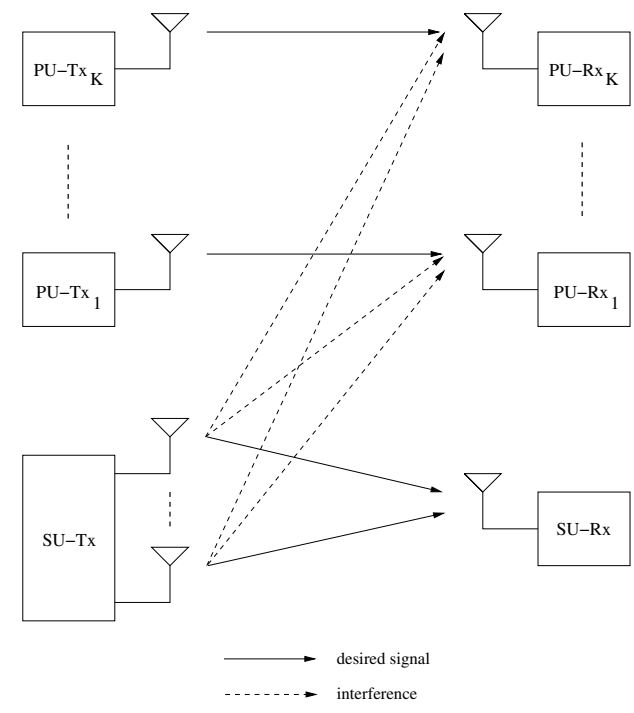

Fig. 1. System Model

of $R \times R$ matrices with complex entries, the real part and the imaginary part. The $j$ th element of vector $\mathbf{h}$ is denoted by $h^{(j)}$. W $\succeq 0$ denotes that $\mathbf{W}$ is positive semidefinite. The notation $\mathrm{x} \sim \mathcal{N}_{\mathcal{C}}(\mathbf{m}, \boldsymbol{\Sigma})$ states that vector $\mathrm{x}$ contains entries of complex Gaussian random variables, with mean $\mathbf{m}$ and covariance $\boldsymbol{\Sigma}$.

\section{SySTEM MOdEL}

As shown in Fig. 1, we consider a cognitive radio system which consists of a secondary transmitter (SU-Tx), a secondary receiver (SU-Rx) and $K$ primary transmitter (PU-Tx) and receiver (PU-Rx) pairs. The SU-Tx has $M$ antennas while there is only one antenna at the SU-Rx and each of the PUTxs and PU-Rxs. We assume that the SU-Tx is located in close proximity of the primary system while the SU-Rx is located at a large distance away from the primary transmitters. Since the PU and SU systems use the same frequency band, the PU-Rxs experience interference from the SU-Tx. In our analysis, we assume that the PU-Tx transmit powers are sufficiently attenuated by distance to be ignored at the SURx. Furthermore, we assume that the PU system employs a time division multiple access (TDMA) scheme, however, due to the loose cooperation between the PU and SU systems, no information regarding PU time slots and synchronisation is available to the SU system. Hence, in our analysis the $\mathrm{SU}$ assumes that all PU-Txs are active simultaneously.

Independent, point-to-point, flat Rayleigh fading channels are assumed for all links in the network. The channel between the $i$ th PU-Tx and the $i$ th PU-Rx and the SU-Tx and $i$ th PU$\mathrm{Rx}$ are denoted by the scalar $c_{i} \in \mathcal{C}$ and the vector $\mathbf{f}_{i} \in \mathcal{C}^{M \times 1}$, respectively, for $i=1 \ldots K . \mathbf{h} \in \mathcal{C}^{M \times 1}$ denotes the channel between the SU-Tx and the SU-Rx. The instantaneous channel powers of these links are represented by $g_{c}^{(i)}=\left|c_{i}\right|^{2}, g_{\mathrm{f}_{i}}^{(j)}=$ $\left|f_{i}^{(j)}\right|^{2}$ and $g_{\mathrm{h}}^{(j)}=\left|h^{(j)}\right|^{2}$ for $j=1 \ldots M$. Furthermore, we assume that the channel powers for all links are independent and identically distributed (i.i.d.) and are governed by their corresponding parameters $\mathbb{E}\left\{g_{c}^{(i)}\right\}=\Omega_{c}^{(i)}, \mathbb{E}\left\{g_{\mathrm{f}_{i}}^{(j)}\right\}=\Omega_{\mathrm{f}_{i}}^{(j)}$ and $\mathbb{E}\left\{g_{\mathrm{h}}^{(j)}\right\}=\Omega_{\mathrm{h}}^{(j)}$.

The signal at the SU-Rx is given by

$$
y=\mathbf{h}^{H} \mathbf{w} s_{\mathrm{s}}+n_{\mathrm{s}},
$$

and that at the $i$ th PU-Rx by

$$
z_{i}=\sqrt{P_{\mathrm{p}}} s_{\mathrm{p}}^{(i)} c_{i}+\mathbf{f}_{i}^{H} \mathbf{w} s_{\mathrm{s}}+n_{\mathrm{p}}^{(i)},
$$

where $s_{\mathrm{S}}$ is information symbol transmitted by the SU-Tx, $\mathbf{w} \in \mathcal{C}^{M \times 1}$ is the beamforming vector at the SU-Tx and $n_{\mathrm{s}}$ is additive white Gaussian noise (AWGN) with variance $\sigma_{\mathrm{s}}^{2}$ at the SU-Rx, $P_{\mathrm{p}}$ is the PU-Tx transmit power, $s_{\mathrm{p}}^{(i)}$ is the information symbol transmitted by the $i$ th PU-Tx and $n_{\mathrm{p}}^{(i)}$ is the AWGN with variance $\sigma_{\mathrm{p}}^{2}$, at the $i$ th PU-Rx. We assume $\mathbb{E}\left\{\left|s_{\mathrm{s}}\right|^{2}\right\}=\mathbb{E}\left\{\left|s_{\mathrm{p}}^{(i)}\right|^{2}\right\}=1$.

The power transmitted by the SU-Tx is given by

$$
P_{T}=\|\mathbf{w}\|^{2} .
$$

Using (1) and (2) and assuming that $s_{\mathrm{S}}$ and $\sqrt{P_{\mathrm{p}}} s_{\mathrm{p}}^{(i)}$, are uncorrelated, the SNR at the SU-Rx can be expressed as

$$
\gamma_{\mathrm{s}}=\frac{\left|\mathbf{h}^{H} \mathbf{w}\right|^{2}}{\sigma_{\mathrm{s}}^{2}},
$$

and the SINR at the $i$ th PU-Rx is given by

$$
\gamma_{\mathrm{p}}^{(i)}=\frac{P_{\mathrm{p}}\left|c_{i}\right|^{2}}{\left|\mathbf{f}_{i}^{H} \mathbf{w}\right|^{2}+\sigma_{\mathrm{p}}^{2}} .
$$

In a cognitive radio system the secondary users are allowed to operate as long as they can guarantee a certain level of quality of service to the primary user. Hence, in our analysis we impose an SINR constraint, $\gamma_{\mathrm{T}}^{(i)}$, at the $i$ th PU-Rx, i.e., $\gamma_{\mathrm{p}}^{(i)} \geq \gamma_{\mathrm{T}}^{(i)}$.

\section{BEAMFORMER OPTIMISATION UNDER FULL CSI}

In this section, we aim to find the optimum beamforming weight vector, w, such that the total SU-Tx transmit power, $P_{T}$, is minimised while guaranteeing minimum QoS to the SU-Rx and each of the PU-Rxs.

In our analysis, we assume that we are unable to control the PU's transmit power and that all of the PUs transmit at a constant power of $P_{\mathrm{p}}$. In this section, we formulate the beamforming problem under the assumption that full CSI for all links is available. This is the basis for the development of the statistically robust beamformer in Section IV.

The total SU-Tx transmit power minimisation problem can be mathematically represented as

$$
\begin{array}{ll}
\min _{\mathbf{w}} & \|\mathbf{w}\| \\
\text { s.t. } & \frac{P_{\mathrm{p}}\left|c_{i}\right|^{2}}{\left|\mathbf{f}_{i}^{H} \mathbf{w}\right|^{2}+\sigma_{\mathrm{p}}^{2}} \geq \gamma_{\mathrm{T}}^{(i)}, \quad i=1 \ldots K \\
& \frac{\left|\mathbf{h}^{H} \mathbf{w}\right|^{2}}{\sigma_{\mathrm{s}}^{2}} \geq \gamma_{\mathrm{s}, \text { min }}
\end{array}
$$

Constraint (6c) is satisfied with equality at the optimum. Otherwise, the optimum $\mathbf{w}$ could be scaled down to satisfy 
the constraint with equality, hence decreasing the objective function and contradicting optimality.

Problem (6) is a non-convex optimisation problem, but it can be reformulated into a convex optimisation problem. Following [7], we observe that neither the objective function nor the constraints change if the beamforming vector undergoes a phase rotation. Thus, $\mathbf{h}^{H} \mathbf{w}$ can be chosen to be real without loss of generality. The transmit power minimisation problem can therefore be restated as the following second order cone program (SOCP)

$$
\begin{array}{ll}
\min _{\mathbf{w}} & \|\mathbf{w}\| \\
\text { s.t. } & \sqrt{P_{\mathrm{p}}\left|c_{i}\right|^{2}} \geq \sqrt{\gamma_{\mathrm{T}}^{(i)}}\left\|\begin{array}{c}
\mathbf{f}_{i}^{H} \mathbf{w} \\
\sigma_{\mathrm{p}}
\end{array}\right\|, \quad i=1 \ldots K \\
& \mathbf{h}^{H} \mathbf{w} \geq \sigma_{\mathrm{s}} \sqrt{\gamma_{\mathrm{s}, \min }} \\
& \Re\left\{\mathbf{h}^{H} \mathbf{w}\right\} \geq 0 \\
& \Im\left\{\mathbf{h}^{H} \mathbf{w}\right\}=0
\end{array}
$$

Problem (7) is in a convex form and standard interior point methods can be used to solve it efficiently.

\section{ROBUST BEAMFORMER OPTIMISATION UNDER PARTIAL AND IMPERFECT CSI}

In practice, full CSI for all links is seldom available and the assumption of full or perfect CSI may be overly idealistic. We consider a CR network with a loose cooperation with the primary network. We assume that only mean channel powers of each of the PU-Tx to PU-Rx and the SU-Tx to each PURx links are available, i.e., only $\Omega_{c}^{(i)} \forall i$ and $\Omega_{\mathrm{f}_{i}}^{(j)} \forall i, j$ for the aforementioned links are available. Furthermore, we assume that the CSI of the SU-Tx to SU-Rx link is imperfect. The imperfection may be due to estimation errors or other factors such as quantisation. In our analysis, we model the CSI errors as additive complex Gaussian noise. Hence

$$
\mathbf{h}=\tilde{\mathbf{h}}+\mathbf{e}
$$

where $\tilde{\mathbf{h}}$ is the imperfect SU-Tx to SU-Rx link CSI estimate known at the SU-Tx and e is the zero mean error vector with i.i.d. complex Gaussian entries and the diagonal covariance matrix $\boldsymbol{\Sigma}_{\mathrm{e}}$, i.e., $\mathbf{e} \sim \mathcal{N}_{\mathcal{C}}\left(\mathbf{0}, \boldsymbol{\Sigma}_{\mathrm{e}}\right)$.

In our formulation we consider the SU and PU outage probability as the QoS parameter. In the system under consideration, outage at the $i$ th PU occurs when its SINR, $\gamma_{\mathrm{p}}^{(i)}$, falls below the threshold $\gamma_{\mathrm{T}}^{(i)}$. Similarly, SU outage occurs when the SU SNR, $\gamma_{\mathrm{s}}$, falls below the SU SNR threshold, $\gamma_{\mathrm{s}, \mathrm{min}}$. The $i$ th PU outage probability is expressed as

$$
\begin{aligned}
\mathrm{P}_{\mathrm{o}}^{(i)} & =\operatorname{Pr}\left\{\gamma_{\mathrm{p}}^{(i)} \leq \gamma_{\mathrm{T}}^{(i)}\right\} \\
& =\operatorname{Pr}\left\{P_{\mathrm{p}}\left|c_{i}\right|^{2}-\gamma_{\mathrm{T}}^{(i)} \mathbf{w}^{H} \mathbf{f}_{i} \mathbf{f}_{i}^{H} \mathbf{w} \leq \gamma_{\mathrm{T}}^{(i)} \sigma_{\mathrm{p}}^{2}\right\},
\end{aligned}
$$

where we have used the relation $\left|\mathbf{f}_{i}^{H} \mathbf{w}\right|^{2}=\mathbf{w}^{H} \mathbf{f}_{i} \mathbf{f}_{i}^{H} \mathbf{w}$. Likewise, the SU outage probability is expressed as

$$
\mathrm{P}_{\mathrm{o}}^{\mathrm{SU}}=\operatorname{Pr}\left\{\left|\mathbf{h}^{H} \mathbf{w}\right|^{2} \leq \gamma_{\mathrm{s}, \min } \sigma_{\mathrm{s}}^{2}\right\}
$$

The probabilistic measures are performed over $c_{i}$ and $\mathbf{f}_{i}$ statistics in (9) and over CSI error statistics in (10).

Hence, given maximum allowable PU and SU outage probabilities, $\alpha^{(i)}$ and $\beta$, constraints (7b) and (7c) are replaced with $\mathrm{P}_{\mathrm{o}}{ }^{(i)} \leq \alpha^{(i)}$ and $\mathrm{P}_{\mathrm{o}}{ }^{\mathrm{SU}} \leq \beta$, respectively.

To proceed, we observe that, in (9), we are dealing with a probability density function (PDF) that is given by the difference of two random variables, namely, $P_{\mathrm{p}}\left|c_{i}\right|^{2}$ and $\gamma_{\mathrm{T}}^{(i)} \mathbf{w}^{H} \mathbf{f}_{i} \mathbf{f}_{i}^{H} \mathbf{w}$. It is easily shown that $P_{\mathrm{p}}\left|c_{i}\right|^{2}$ has an exponential distribution with a mean of $P_{\mathrm{p}} \Omega_{c}^{(i)}$. The probability distribution of $\gamma_{\mathrm{T}}^{(i)} \mathbf{w}^{H} \mathbf{f}_{i} \mathbf{f}_{i}^{H} \mathbf{w}$ can be found using the following lemma.

Lemma 1. If $\mathbf{x} \in \mathcal{C}^{R \times 1}$ is distributed as $\mathbf{x} \sim \mathcal{N}_{\mathcal{C}}(\mathbf{0}, \boldsymbol{\Sigma})$, then for any deterministic positive semidefinite Hermitian matrix $\mathbf{A} \in \mathcal{C}^{R \times R}$, the PDF of the random variable $\psi=\mathbf{x}^{H} \mathbf{A x}$, $\psi \geq 0$, is given by

$$
f(\psi)=\left[\prod_{i=1}^{N} \lambda_{i}\right] \sum_{j=1}^{N} \frac{\exp \left(-\lambda_{j} \psi\right)}{\prod_{k=1, k \neq j}^{N}\left(\lambda_{k}-\lambda_{j}\right)},
$$

where $\lambda_{i}=1 / \Lambda_{i}$, and $\Lambda_{i}, i=1 \ldots N \leq R$ are the non-zero eigenvalues of $\boldsymbol{\Sigma} \mathbf{A}$. Note that this is precisely the distribution of the sum of $N$ exponentially distributed independent random variables, each with a mean of $\Lambda_{i}$.

Proof: Note that $\mathbf{x}^{H} \mathbf{A x}$ is commonly known as a quadratic form in normal random variables [21]. We first define $\mathbf{y}=\boldsymbol{\Sigma}^{-\frac{1}{2}} \mathbf{X}$. It is easily verified that $\mathbf{y} \sim \mathcal{N}_{\mathcal{C}}(\mathbf{0}, \mathbf{I})$. Using an orthogonal $R \times R$ matrix $\mathbf{P}$ that diagonalises $\boldsymbol{\Sigma}^{\frac{1}{2}} \mathbf{A} \boldsymbol{\Sigma}^{\frac{1}{2}}$ or equivalently $\boldsymbol{\Sigma} \mathbf{A}$, i.e., $\mathbf{P}^{H} \boldsymbol{\Sigma} \mathbf{A P}=\operatorname{diag}\left(\Lambda_{1}, \Lambda_{2}, \ldots, \Lambda_{R}\right)$ and $\Lambda_{1}, \Lambda_{2}, \ldots, \Lambda_{R}$ are the eigenvalues of $\boldsymbol{\Sigma} \mathbf{A}$. $\psi$ can then be expressed as

$$
\begin{aligned}
\psi & =\mathbf{y}^{H} \boldsymbol{\Sigma}^{\frac{1}{2}} \mathbf{A} \boldsymbol{\Sigma}^{\frac{1}{2}} \mathbf{y}=\left(\mathbf{P}^{H} \mathbf{y}\right)^{H} \mathbf{P}^{H} \boldsymbol{\Sigma}^{\frac{1}{2}} \mathbf{A} \boldsymbol{\Sigma}^{\frac{1}{2}} \mathbf{P}\left(\mathbf{P}^{H} \mathbf{y}\right) \\
& =\operatorname{tr}\left(\operatorname{diag}\left(\Lambda_{1}, \Lambda_{2}, \ldots, \Lambda_{R}\right) \mathbf{P}^{H} \mathbf{y} \mathbf{y}^{H} \mathbf{P}\right) \\
& =\sum_{i=1}^{N} \Lambda_{i}\left|\left(\mathbf{P}^{H} \mathbf{y}\right)_{i}\right|^{2}
\end{aligned}
$$

where $N \leq R$ is the number of non-zero eigenvalues of $\boldsymbol{\Sigma} \mathbf{A}$ and $\left(\mathbf{P}^{H} \mathbf{y}\right)_{i}$ is the $i$ th element of the vector $\mathbf{P}^{H} \mathbf{y}$. Since $\mathbf{P}$ is an orthogonal matrix, it is easily shown that $\mathbf{P}^{H} \mathbf{y} \sim \mathcal{N}_{\mathcal{C}}(\mathbf{0}, \mathbf{I})$, and therefore, $\left|\left(\mathbf{P}^{H} \mathbf{y}\right)_{i}\right|^{2}$ is an exponentially distributed random variable with a mean of $\Lambda_{i}$. Hence, (12) is a sum of $N$ exponentially distributed independent random variables, each with a mean of $\Lambda_{i}$, whose PDF is given by (11). The derivation of the PDF of the sum of $N$ exponentially distributed independent random variables has appeared in many texts, we refer the interested reader to [22] and references therein.

We note that $\gamma_{\mathrm{T}}^{(i)} \mathbf{w}^{H} \mathbf{f}_{i} \mathbf{f}_{i}^{H} \mathbf{w}=\gamma_{\mathrm{T}}^{(i)} \mathbf{f}_{i}^{H} \mathbf{W} \mathbf{f}_{i}$, where $\mathbf{W}=$ $\mathbf{w} \mathbf{w}^{H}$. Using Lemma 1 and exploiting the fact that $\mathbf{W}$ is a rank-one matrix, we have that $\gamma_{\mathrm{T}}^{(i)} \mathbf{w}^{H} \mathbf{f}_{i} \mathbf{f}_{i}^{H} \mathbf{w}$ is exponentially distributed with a mean of $\gamma_{\mathrm{T}}^{(i)} \operatorname{tr}\left(\boldsymbol{\Sigma}_{\mathbf{f}_{i}} \mathbf{W}\right)$, where $\boldsymbol{\Sigma}_{\mathbf{f}_{i}}$ is the covariance matrix of $\mathbf{f}_{i}$ and is expressed as

$$
\boldsymbol{\Sigma}_{\mathbf{f}_{i}}=\operatorname{diag}\left(\boldsymbol{\Omega}_{\mathrm{f}_{i}}\right),
$$


where $\Omega_{\mathrm{f}_{i}}=\left[\Omega_{\mathrm{f}_{i}}^{(1)} \Omega_{\mathrm{f}_{i}}^{(2)} \ldots \Omega_{\mathrm{f}_{i}}^{(M)}\right]^{T}$.

The PDF in (9) is that of a difference between two independent exponential random variables and can easily be shown to have the following form

$$
f(\psi)= \begin{cases}\frac{\lambda_{1} \lambda_{2}}{\lambda_{1}+\lambda_{2}} \exp \left(-\lambda_{1} \psi\right) & \text { if } \psi \geq 0, \\ \frac{\lambda_{1} \lambda_{2}}{\lambda_{1}+\lambda_{2}} \exp \left(\lambda_{2} \psi\right) & \text { if } \psi<0,\end{cases}
$$

where $\lambda_{1}=1 /\left(P_{\mathrm{p}} \Omega_{c}^{(i)}\right)$ and $\lambda_{2}=1 /\left(\gamma_{\mathrm{T}}^{(i)} \operatorname{tr}\left(\boldsymbol{\Sigma}_{\mathbf{f}_{i}} \mathbf{W}\right)\right)$.

Using (14) and utilising the fact that $\gamma_{\mathrm{T}}^{(i)} \sigma_{\mathrm{p}}^{2} \geq 0$, (9) can be rewritten as

$$
\begin{aligned}
\mathrm{P}_{\mathrm{o}}{ }^{(i)} & =1-\int_{\gamma_{\mathrm{T}}^{(i)} \sigma_{\mathrm{p}}^{2}}^{\infty} \frac{\lambda_{1} \lambda_{2}}{\lambda_{1}+\lambda_{2}} \exp \left(-\lambda_{1} \psi\right) \mathrm{d} \psi \\
& =1-\frac{\lambda_{2}}{\lambda_{1}+\lambda_{2}} \exp \left(-\lambda_{1} \gamma_{\mathrm{T}}^{(i)} \sigma_{\mathrm{p}}^{2}\right) .
\end{aligned}
$$

Hence, the $i$ th PU outage probability constraint can be written as the following convex constraint

$$
\operatorname{tr}\left(\boldsymbol{\Sigma}_{\mathbf{f}_{i}} \mathbf{W}\right) \leq \frac{P_{\mathrm{p}} \Omega_{c}^{(i)}\left(\exp \left(-\frac{\gamma_{\mathrm{T}}^{(i)} \sigma_{\mathrm{p}}^{2}}{P_{\mathrm{p}} \Omega_{c}^{(i)}}\right)-1+\alpha^{(i)}\right)}{\gamma_{\mathrm{T}}^{(i)}\left(1-\alpha^{(i)}\right)} .
$$

An important observation in the above constraint is that it is dependent only on the diagonal elements of $\mathbf{W}$, i.e., dependent only on the beamformer transmit power. This is a fairly intuitive result since phase information of the SU-Tx to PU-Rx links are not available and, therefore, power control is the only degree of freedom available to the beamformer to control the amount of interference to the PU-Rxs.

In (10), $\left|\mathbf{h}^{H} \mathbf{w}\right|^{2}$ is recognised to be a non-central Chisquare random variable whose $\mathrm{CDF}$ is given by the generalised Marcum's Q function [23]. The generalised Marcum's Q function is difficult to handle and so to obtain a mathematically tractable solution, we rewrite (10) as follows

$$
\begin{gathered}
\mathrm{P}_{\mathrm{o}}^{\mathrm{SU}}=\operatorname{Pr}\left\{\mathbf{w}^{H} \tilde{\mathbf{h}} \tilde{\mathbf{h}}^{H} \mathbf{w}+\mathbf{w}^{H}\left(\tilde{\mathbf{h}} \mathbf{e}^{H}+\mathbf{e h}^{H}\right) \mathbf{w}\right. \\
\quad+\mathbf{w}^{H} \mathbf{e} \mathbf{e}^{H} \mathbf{w} \leq \gamma_{\left.\mathbf{s}, \min \sigma_{\mathrm{s}}^{2}\right\}} \underbrace{}_{u} \underbrace{2 \Re\left\{\mathbf{w}^{H} \tilde{\mathbf{h}} \mathbf{e}^{H} \mathbf{w}\right\}}_{v}+\underbrace{\mathbf{w}^{H} \mathbf{e e}^{H} \mathbf{w}}_{u} \\
\left.\leq \gamma_{\mathrm{s}, \min } \sigma_{\mathrm{s}}^{2}-\mathbf{w}^{H} \tilde{\mathbf{h}} \tilde{\mathbf{h}}^{H} \mathbf{w}\right\} .
\end{gathered}
$$

Since $u$ in (17) is a linear combination of zero-mean independent Gaussian random variables, it itself is a zero-mean Gaussian random variable with variance $\sigma_{u}^{2}$ given by

$$
\begin{aligned}
\sigma_{u}^{2} & =2 \mathbb{E}\left\{\mathbf{w}^{H} \tilde{\mathbf{h}} \mathbf{e}^{H} \mathbf{w} \mathbf{w}^{H} \mathbf{e} \tilde{\mathbf{h}}^{H} \mathbf{w}\right\} \\
& =2 \mathbf{w}^{H} \tilde{\mathbf{h}} \mathbf{w}^{H} \mathbb{E}\left\{\mathbf{e} \mathbf{e}^{H}\right\} \mathbf{w} \tilde{\mathbf{h}}^{H} \mathbf{w} \\
& =2 \operatorname{tr}\left(\tilde{\mathbf{h}} \tilde{\mathbf{h}}^{H} \mathbf{W}\right) \operatorname{tr}\left(\boldsymbol{\Sigma}_{\mathrm{e}} \mathbf{W}\right) \\
& =2 \operatorname{tr}\left(\tilde{\mathbf{h}} \tilde{\mathbf{h}}^{H} \mathbf{W} \boldsymbol{\Sigma}_{\mathrm{e}} \mathbf{W}\right) \\
& =2\left\|\left(\boldsymbol{\Sigma}_{\mathrm{e}} \tilde{\mathbf{h}} \tilde{\mathbf{h}}^{H}\right)^{\frac{1}{2}} \mathbf{W}\right\|^{2},
\end{aligned}
$$

where $\mathbf{W}=\mathbf{w w}^{H}$. Using Lemma $1, v$ in (17) is recognised as an exponentially distributed random variable with mean $\mu_{v}=$ $\operatorname{tr}\left(\boldsymbol{\Sigma}_{\mathrm{e}} \mathbf{W}\right)$ and variance $\sigma_{v}^{2}=\operatorname{tr}\left(\boldsymbol{\Sigma}_{\mathrm{e}} \mathbf{W}\right)^{2}$.

When channel uncertainty is large, beamforming loses its effectiveness since the beam width has to be widened to handle the channel uncertainty. In this paper we focus on the scenario where the channel uncertainty is in the acceptable range for beamforming to be practical. In this scenario, $\sigma_{v}^{2}$ is much smaller than $\sigma_{u}^{2}$ and as a result the PDF in (17) is dominated by the Gaussian random variable $v$. Therefore, the PDF in (17) can be approximated by the zero-mean Gaussian distribution with variance $\sigma_{u}^{2}$. Fig. 2 compares the true distribution with its Gaussian approximation for two values of channel error variance. Channel error is modelled as $\mathbf{e} \sim \mathcal{N}_{\mathcal{C}}\left(\mathbf{0}, \sigma_{e}^{2} \mathbf{I}\right)$, i.e., $\boldsymbol{\Sigma}_{\mathrm{e}}=\sigma_{e}^{2} \mathbf{I}$.

The SU outage probability can therefore be approximated as

$$
\mathrm{P}_{\mathrm{o}} \mathrm{SU} \approx 1-\frac{1}{2} \operatorname{erfc}\left(\frac{\gamma_{\mathrm{s}, \min } \sigma_{\mathrm{s}}^{2}-\operatorname{tr}\left(\tilde{\mathbf{h}} \tilde{\mathbf{h}}^{H} \mathbf{W}\right)}{2\left\|\left(\boldsymbol{\Sigma}_{\mathrm{e}} \tilde{\mathbf{h}} \tilde{\mathbf{h}}^{H}\right)^{\frac{1}{2}} \mathbf{W}\right\|}\right),
$$

and the SU outage probability constraint can be expressed as the convex constraint

$$
\frac{\gamma_{\mathrm{s}, \min } \sigma_{\mathrm{s}}^{2}-\operatorname{tr}\left(\tilde{\mathbf{h}} \tilde{\mathbf{h}}^{H} \mathbf{W}\right)}{\operatorname{erfc}^{-1}(2(1-\beta))} \geq 2\left\|\left(\boldsymbol{\Sigma}_{\mathrm{e}} \tilde{\mathbf{h}} \tilde{\mathbf{h}}^{H}\right)^{\frac{1}{2}} \mathbf{W}\right\| .
$$

The robust transmit power minimisation problem can therefore be stated as the following semidefinite program (SDP) [24]

$$
\begin{array}{cl}
\min _{\mathbf{W}} & \operatorname{tr}(\mathbf{W}) \\
\text { s.t. } & (16), \quad i=1 \ldots K \\
& (20) \\
& \mathbf{W} \succeq 0 \\
& \operatorname{rank}(\mathbf{W})=1
\end{array}
$$

Constraints (21d) and (21e) are included in the above optimisation problem because of the definition of $\mathbf{W}$. Note that constraint (21e) is a non-convex constraint hence, we apply the idea of semidefinite relaxation (SDR) $[24,25]$ and relax problem (21) by dropping the non-convex rank-one constraint and obtain the following convex robust transmit power minimisation SDP

$$
\begin{array}{ll}
\min _{\mathbf{W}} & \operatorname{tr}(\mathbf{W}) \\
\text { s.t. } & (16), \quad i=1 \ldots K \\
& (20) \\
& \mathbf{W} \succeq 0
\end{array}
$$

Problem (22) is in a convex form and standard interior point methods can be used to solve it efficiently.

After solving (22) one needs to recover the optimum beamforming vector, $\mathbf{w}^{*}$, from $\mathbf{W}$. In [26] it was shown that, in beamforming problems of this nature, the solution obtained is always rank-one and, therefore, $\mathbf{w}^{*}$ can be chosen to be the principle eigenvector of $\mathbf{W}$. In our extensive numerical simulations we have never obtained a solution that had a rank higher than one. 


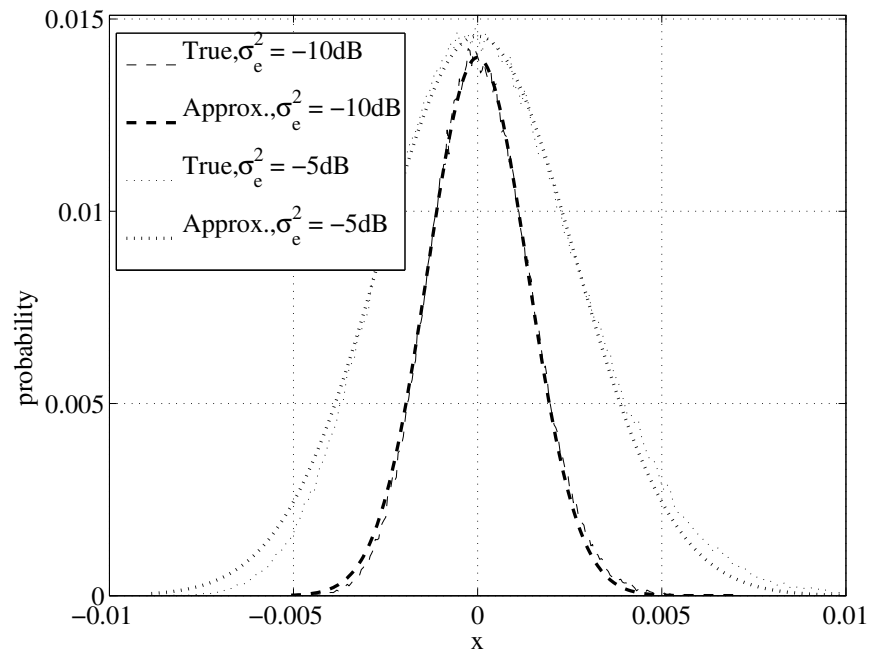

Fig. 2. True PDF and Approximation

\section{Simulation Results and Discussion}

We illustrate the performance of our proposed methods through numerical simulations in i.i.d. Rayleigh flat-fading channels. We consider a system with four PUs and one SU with four transmit antennas, i.e., $K=4$ and $M=4$. In all simulations we have set $P_{\mathrm{p}}=30 \mathrm{dBm}, \gamma_{\mathrm{T}}^{(i)}=10 \mathrm{~dB}$, $\gamma_{\mathrm{s}, \min }=10 \mathrm{~dB}$ and the noise power at each PU and SU receiver is assumed to be $-5 \mathrm{dBm}$, i.e., $\sigma_{\mathrm{p}}^{2}=\sigma_{\mathrm{s}}^{2}=-5$ $\mathrm{dBm}$. The maximum PU outage probability, $\alpha^{(i)}$, is set to $20 \%$. Similarly, the maximum SU outage probability, $\beta$ is also set to $20 \%$. Channel powers in all links are set to 0 $\mathrm{dB}$, i.e., $\Omega_{c}^{(i)}=0 \mathrm{~dB}, \Omega_{\mathrm{f}_{i}}^{(j)}=0 \mathrm{~dB}$ and $\Omega_{\mathrm{h}}^{(j)}=0 \mathrm{~dB}$. The SU channel error is modelled as $\mathbf{e} \sim \mathcal{N}_{\mathcal{C}}\left(\mathbf{0}, \sigma_{e}^{2} \mathbf{I}\right)$, i.e, $\boldsymbol{\Sigma}_{\mathrm{e}}=\sigma_{e}^{2} \mathbf{I}$. The error variance, $\sigma_{e}^{2}$, is referenced from the mean SU channel power and simulations are performed for two values of $\sigma_{e}^{2}$, specifically, $-10 \mathrm{~dB}$ and $-5 \mathrm{~dB}$. Results of our proposed method are compared against the full CSI, worst case and nonrobust designs.

The worst case beamformer is designed such that the SINR at the $i$ th PU-Rx is above the threshold $\gamma_{\mathrm{T}}^{(i)}$ and the SNR at the SU-Rx is above the threshold $\gamma_{\mathrm{s}, \min }$ for every possible realisation of $c_{i}, \mathbf{f}_{i}$ and $\mathbf{e}$. Since instantaneous realisations of $c_{i}$ and $\mathbf{f}_{i}$ are not available, our worst case design solves problem (6) based on the expected value of (5). Note that (5) is at its minimum when $\left|c_{i}\right|^{2}=\Omega_{c}^{(i)}-\epsilon_{c}$ and $\left|f_{i}^{(j)}\right|^{2}=\Omega_{\mathrm{f}_{i}}^{(j)}+\epsilon_{f}$, for some appropriately chosen values of $\epsilon_{c}, \epsilon_{f} \geq 0$. The worst case beamformer ensures this minimum value is always above the threshold $\gamma_{\mathrm{T}}^{(i)}$. Furthermore, we impose a norm bound [27] on e, i.e., $\|\mathbf{e}\| \leq \epsilon_{e}$ and the worst case beamformer ensures that the SNR at the SU-Rx is above the threshold $\gamma_{\mathrm{s}, \min }$ for every possible realisation of e within this bound. To provide a fair comparison with the method proposed in this paper, $\epsilon_{c}, \epsilon_{f}$ and $\epsilon_{e}$ are chosen such that $\operatorname{Pr}\left\{\left|c_{i}\right|^{2} \geq \Omega_{c}^{(i)}-\epsilon_{c}\right\} \prod_{j=1}^{R} \operatorname{Pr}\left\{\left|f_{i}^{(j)}\right|^{2} \leq \Omega_{\mathrm{f}_{i}}^{(j)}+\epsilon_{f}\right\}=$ $1-\alpha^{(i)}, \forall i$ and $\operatorname{Pr}\left\{\|\mathbf{e}\| \leq \epsilon_{e}\right\}=1-\beta$.
In Fig. 3, results are provided for the CDF of the SINR at the first PU (PU 1). We see that the required probability of the resulting PU-Rx SINR being below $10 \mathrm{~dB}$ is satisfied by the robust optimisation scheme proposed in this paper for both error variance values. The other methods also achieve the required performance. The full CSI design achieves the best performance, while the worst case design results in the worst performance. This is because the PU and SU systems have conflicting requirements and, as the performance of the $\mathrm{SU}$ in terms of achieved SNR increases, it comes at a loss of performance of the PU.

The SU-Rx SNR and the SU-Tx transmit power CDFs for the proposed method along with the full CSI, non-robust and the worst case designs are shown in Figs. 4 and 5, respectively. We see that the probability of outage for the full CSI design is zero and it achieves this by utilising the least amount of transmit power. The full CSI design performance serves as a benchmark for the other methods. The proposed robust beamformer satisfies the outage probability constraint for both error variance values. We observe that as the channel error power increases the SNR curves move away from the ideal step function response of the full CSI case. We also see that the proposed beamformer utilises more transmit power than the full CSI design and the power consumption increases as the channel error variance increases. This is because the robust beamformer has to deal with more uncertainty in the channel and, in doing so, consumes more transmit power. The probability that the SNR is below $10 \mathrm{~dB}$ in the worst case approach is almost zero. The worst case design SNR curve is the furthest away from the ideal scenario and this design also consumes the largest amount of transmit power. This is because the beamformer is optimised to protect the PU-Rx on every possible CSI realisation in the chosen set. Although the non-robust method utilises the same amount of power as the full CSI design, the outage probability is almost $50 \%$ because the outage probability constraint is not respected. To summarise, our results reveal that the statistically robust beamformer proposed in this paper provides the best performance when channel uncertainties exist.

\section{CONCLUSIONS}

In this paper, we have studied a statistically robust beamformer for a CR network under the assumption of partial and imperfect CSI. We have shown that the robust SU transmit power minimisation problem can be formulated as a convex optimisation problem using probabilistic constraints. Our results reveal that the robust beamformer proposed in this paper provides the best performance when channel uncertainties exist.

\section{REFERENCES}

[1] P. A. Dmochowski, H. A. Suraweera, P. J. Smith, and M. Shafi, "Impact of channel knowledge on cognitive radio system capacity," in Proc. IEEE VTC2010-Fall, Sep. 2010, pp. 1-5.

[2] M. Shafi, H. A. Suraweera, P. J. Smith, and M. Faulkner, "Capacity limits and performance analysis of cognitive radio with imperfect channel knowledge," IEEE Trans. on Veh. Technol., Jan. 2010. 


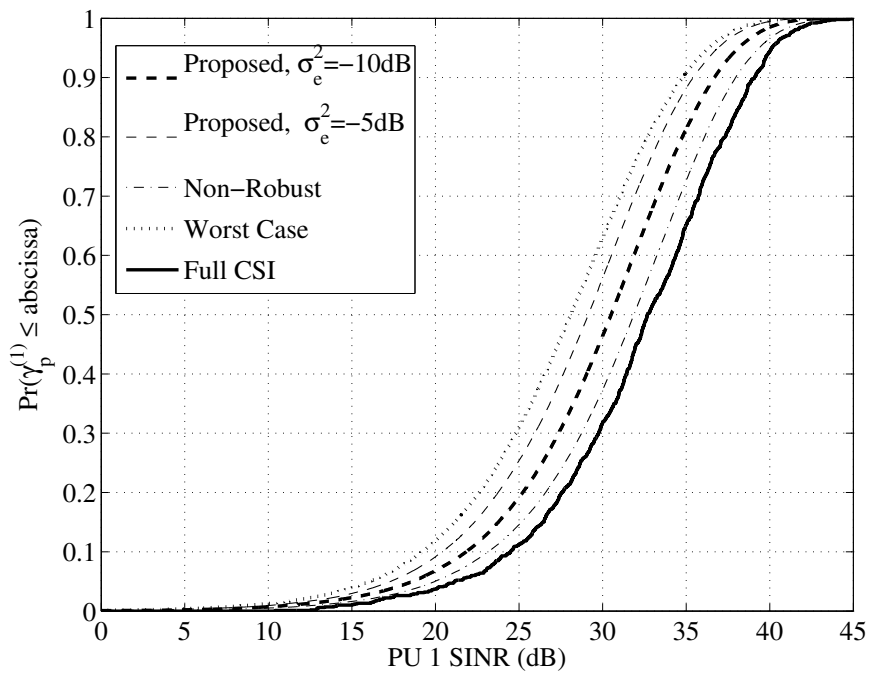

Fig. 3. CDF of SINR at PU 1

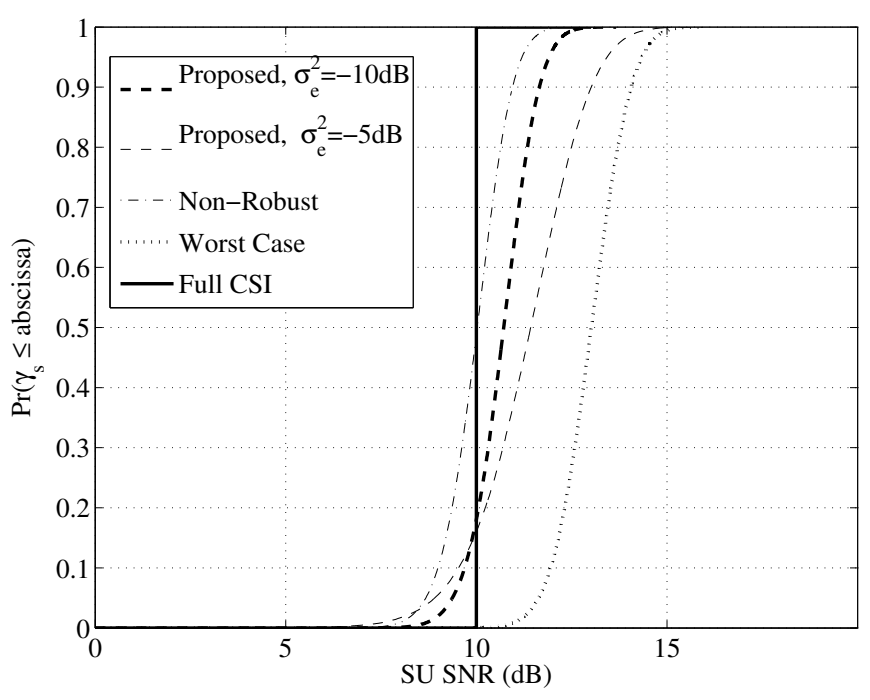

Fig. 4. CDF of SNR at the SU

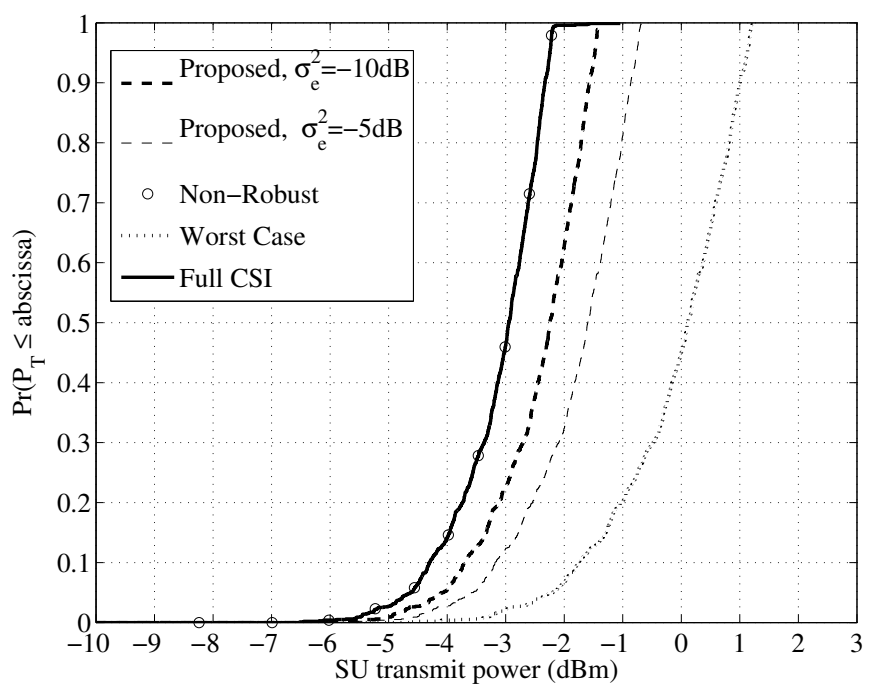

Fig. 5. CDF of total SU transmit power
[3] F. Rashid-Farrokhi, K. Liu, and L. Tassiulas, "Transmit beamforming and power control for cellular wireless systems," IEEE J. Sel. Areas in Commun., vol. 16, no. 8, pp. 1437 -1450, Oct. 1998.

[4] F. Rashid-Farrokhi, L. Tassiulas, and K. Liu, "Joint optimal power control and beamforming in wireless networks using antenna arrays," IEEE Trans. Commun., vol. 46, no. 10, pp. 1313 -1324, Oct. 1998.

[5] E. Visotsky and U. Madhow, "Optimum beamforming using transmit antenna arrays," in Proc. IEEE Veh. Tech. Conf., vol. 1, Jul. 1999, pp. 851-856.

[6] M. Schubert and H. Boche, "Solution of the multiuser downlink beamforming problem with individual sinr constraints," IEEE Trans. Veh. Tech., vol. 53, no. 1, pp. 18 - 28, Jan. 2004.

[7] M. Bengtsson and B. Ottersten, Handbook on Antennas in Wireless Communications. CRC, 2002, ch. Optimal and suboptimal transmit beamforming.

[8] A. Wiesel, Y. Eldar, and S. Shamai, "Linear precoding via conic optimization for fixed MIMO receivers," IEEE Trans. Signal Process., vol. 54, no. 1, pp. 161 - 176, Jan. 2006.

[9] Y. Nesterov and A. Nemirovski, Interior Point Polynomial Methods in Convex Programming. SIAM Press, 1994.

[10] A. Gershman, Z. Luo, S. Shahbazpanahi, and S. Vorobyov, "Robust adaptive beamforming using worst-case performance optimization," in 37th Asilomar Conf. on Signals, Systems and Computers, vol. 2, Nov. 2003, pp. 1353-1357.

[11] S. Shahbazpanahi, A. Gershman, Z. Luo, and K. M. Wong, "Robust adaptive beamforming for general-rank signal models," IEEE Trans. Signal Process., vol. 51, no. 9, pp. 2257 - 2269, Sep. 2003.

[12] S. Kim, A. Magnani, A. Mutapcic, S. Boyd, and Z. Luo, "Robust beamforming via worst-case sinr maximization," IEEE Trans. Signal Process., vol. 56, no. 4, pp. 1539 -1547, Apr. 2008

[13] A. El-Keyi, T. Kirubarajan, and A. Gershman, "Wideband robust beamforming based on worst-case performance optimization," in IEEE 13th Workshop on Statistical Signal Process., Jul. 2005, pp. 265 -270.

[14] B. K. Chalise, S. Shahbazpanahi, A. Czylwik, and A. B. Gershman, "Robust downlink beamforming based on outage probability specifications," IEEE Trans. Wireless Commun., vol. 6, no. 10, pp. 3498-3503, Oct. 2007.

[15] M. Shenouda and T. Davidson, "Probabilistically-constrained approaches to the design of the multiple antenna downlink," in Proc. 42nd Asilomar Conf. Signals, Systems and Computers, Oct. 2008, pp. $1120-1124$.

[16] L. Zhang, Y. Liang, and Y. Xin, "Joint beamforming and power allocation for multiple access channels in cognitive radio networks," IEEE J. Select. Areas Commun., vol. 26, no. 1, pp. 38 -51, Jan. 2008.

[17] R. Zhang and Y. Liang, "Exploiting multi-antennas for opportunistic spectrum sharing in cognitive radio networks," IEEE J. Select. Topics Signal Process., vol. 2, no. 1, pp. 88 -102, Feb. 2008.

[18] L. Zhang, Y. Liang, Y. Xin, and H. Poor, "Robust cognitive beamforming with partial channel state information," IEEE Trans. Wireless Commun., vol. 8, no. 8, pp. $4143-4153$, Aug. 2009.

[19] G. Zheng, K. Wong, A. Paulraj, and B. Ottersten, "Robust collaborativerelay beamforming," IEEE Trans. Sig. Process., vol. 57, no. 8, pp. 3130 -3143 , Aug. 2009.

[20] G. Zheng, S. Ma, K. Wong, and T. Ng, "Robust beamforming in cognitive radio," IEEE Trans. Wireless Commun., vol. 9, no. 2, pp. 570 -576 , Feb. 2010.

[21] A. M. Mathai and S. B. Provost, Quadratic Forms In Random Variables, Theory and Applications. Marcel Dekker, INC., 1992.

[22] S. Kandukuri and S. Boyd, "Optimal power control in interference limited fading wireless channels with outage probability specifications," IEEE Trans. Wireless Commun., vol. 1, no. 1, pp. 46-55, Jan. 2002.

[23] J. G. Proakis, Digital Communications, 4th ed. McGraw-Hill, 2001.

[24] S. Boyd and L. Vandenberghe, Convex Optimization. Cambridge University Press, 2009.

[25] Z. Luo and T. Chang, Convex Optimization in Signal Processing and Communications. Cambridge University Press, 2010, ch. 4 SDP relaxation of homogeneous quadratic optimization: approximation bounds and applications, pp. 117-165.

[26] A. B. Gershman, N. D. Sidiropoulos, S. Shahbazpanahi, M. Bengtsson, and B. Ottersten, "Convex optimization based beamforming," IEEE Signal Proc. Mag., vol. 27, no. 3, pp. 62-75, May 2010.

[27] S. Vorobyov, A. Gershman, and Z. Luo, "Robust adaptive beamforming using worst-case performance optimization: a solution to the signal mismatch problem," IEEE Trans. Signal Process., vol. 51, no. 2, pp. 313 - 324, Feb. 2003. 